\title{
High-flow nasal cannula for Acute Respiratory Distress Syndrome (ARDS) due to COVID-19
}

\author{
Carolina Panadero, ${ }^{1}$ Araceli Abad-Fernández, ${ }^{1} \mathrm{M}^{\mathrm{a}}$ Teresa Rio-Ramirez, ${ }^{1}$ Carmen Maria Acosta Gutierrez, ${ }^{1}$ Mariara \\ Calderon-Alcala, ${ }^{1}$ Cristina Lopez-Riolobos, ${ }^{1}$ Cristina Matesanz-Lopez, ${ }^{1}$ Fernando Garcia-Prieto, ${ }^{1}$ Jose Maria Diaz- \\ Garcia, ${ }^{1}$ Beatriz Raboso-Moreno, ${ }^{1}$ Zully Vasquez-Gambasica, ${ }^{1}$ Pilar Andres-Ruzafa, ${ }^{1}$ Jose Luis Garcia-Satue, ${ }^{1}$ \\ Sara Calero-Pardo, ${ }^{1}$ Belen Sagastizabal, ${ }^{2}$ Diego Bautista, ${ }^{2}$ Alfonso Campos, ${ }^{2}$ Marina González, ${ }^{2}$ Luis Grande, ${ }^{2}$ \\ Marta Jimenez Fernandez, ${ }^{3}$ Jose L. Santiago-Ruiz, ${ }^{4}$ Pedro Caravaca Perez, ${ }^{5}$ Andres Jose Alcaraz ${ }^{2}$ \\ ${ }^{1}$ Pulmonology Department, Hospital Universitario de Getafe, Madrid \\ ${ }^{2}$ Department of Pediatrics, Hospital Universitario de Getafe, Madrid \\ ${ }^{3}$ Thoracic Surgery Department, Hospital Universitario de Getafe, Madrid \\ ${ }^{4}$ Cardiology Department, Hospital Universitario de Getafe, Madrid \\ ${ }^{5}$ Cardiology Department, Hospital Universitario 12 de Octubre, Instituto de Investigación Sanitaria Hospital 12 de Octubre \\ (imas12), CIBERCV, Madrid, Spain
}

\begin{abstract}
Introduction: High-flow nasal cannula oxygen therapy (HFNC) has been shown to be a useful therapy in the treatment of patients with Acute Respiratory Distress Syndrome (ARDS), but its efficacy is still unknown in patients with COVID-19. Our objective is to describe its utility as therapy for the treatment of ARDS caused by SARS-CoV-2.

Methods: A retrospective, observational study was performed at a single centre, evaluating patients with ARDS secondary to COVID-19 treated with HFNC. The main outcome was the intubation rate at day 30, which defined failure of therapy. We also analysed the role of the ROX index to predict the need for intubation.

Results: In the study period, 196 patients with bilateral pneumonia were admitted to our pulmonology unit, 40 of whom were treated with HFNC due to the presence of ARDS. The intubation rate at day 30 was $52.5 \%$, and overall mortality was $22.5 \%$. After initiating $\mathrm{HFNC}$, the $\mathrm{SpO}_{2} / \mathrm{FiO}_{2}$ ratio was significantly better in the group that did not require intubation $(113.4 \pm 6.6 v s 93.7 \pm 6.7, \mathrm{p}=0.020)$, as was the ROX index $(5.0 \pm 1.6 v s 4.0 \pm 1.0, \mathrm{p}=0.018)$. A ROX index less than 4.94 measured 2 to $6 \mathrm{~h}$ after the start of therapy was associated with increased risk of intubation (HR 4.03 [95\% CI 1.18 - 13.7]; $\mathrm{p}=0.026$ ).

Conclusion: High-flow therapy is a useful treatment in ARDS in order to avoid intubation or as a bridge therapy, and no increased mortality was observed secondary to the delay in intubation. After initiating HFNC, a ROX index below 4.94 predicts the need for intubation.
\end{abstract}

Key words: COVID-19; high-flow nasal cannula; acute respiratory distress syndrome; coronavirus.

Correspondence: Carolina Panadero, Pulmonology Department, Hospital Universitario de Getafe, Carr. Madrid-Toledo km 12.500, 28905 Getafe, Madrid, Spain. E-mail: carolina.panadero@salud.madrid.org

Contributions: $\mathrm{CP}$, designed the study, contributed to the acquisition, analysis, and interpretation of data, wrote the manuscript and revised the manuscript; AA, contributed to the acquisition of data, wrote the manuscript and revised the manuscript; MTRR, CMAG, MCA, CLR, CML, FGP, JMDG, BRM, ZVG, PAR, JLGS, SCP, BS, DB, AC, MG, LG, MJF, JLS, AJA contributed to the acquisition of data; PCP, contributed to the analysis, and interpretation of data and revised the manuscript. All the authors made a substantive intellectual contribution, read and approved the final version of the manuscript and agreed to be accountable for all aspects of the work.

Conflict of interest: The authors have no competing interests.

Availability of data and materials: Study data analyzed will be made available from the corresponding author on reasonable request.

Ethics approval and consent to participate: This study has obtained a Favourable Report from the Ethics Committee for Research with Medicines (CEIm) of the Hospital Universitario de Getafe. Given the retrospective nature of the study, no informed consent was collected.

Consent for publication: Not applicable. 


\section{Introduction}

COVID-19 disease caused by the SARS-CoV-2 coronavirus began in Wuhan, China in December 2019, and subsequently spread around the world [1]. Up to $20 \%$ of patients develop acute respiratory distress syndrome (ARDS) [2], which may require ventilatory support and intensive care unit (ICU) admission. During the pandemic, the health system in Madrid (Spain) was overwhelmed, so access to critical care units became limited due to the high demand. Motivated by this exceptional situation, non-invasive respiratory support has been optimised in order to avoid or delay admission to the ICU, especially in times of high saturation. High-flow nasal cannula oxygen therapy (HFNC) [3] is a respiratory support modality that supplies high levels of oxygen through a nasal cannula, providing active humidification and high flows that reduce dead space, improve $\mathrm{CO}_{2}$ clearance, and provide low levels of positive pressure [4]. It has been shown to be a useful therapy in the treatment of ARDS and is better tolerated than other non-invasive ventilation (NIV) methods. Compared to invasive mechanical ventilation, it has the advantages of producing less ventilator-induced lung injury (VILI) [5], while avoiding the nosocomial complications associated with intubation. In contrast, HFNC is a potential aerosol-generating technique, with the consequent risk of infection. Furthermore, some studies have observed increased mortality in patients with delayed intubation.

Presently, there is no solid evidence about the benefits and risks of using HFNC for ARDS due to SARS-CoV-2. Thus, there are no clear recommendations on its use, and it is not clear whether HFNC is an effective therapy. Our study aims to describe the experience of using high-flow oxygen therapy in an intermediate respiratory care unit during the COVID-19 pandemic, focusing on its usefulness in preventing intubation in patients with ARDS and determining the factors associated with failure of the therapy.

\section{Methods}

\section{Study population}

A retrospective, observational study was conducted in the Intermediate Respiratory Care Unit (IRCU) of the Pulmonology Department at the Hospital Universitario de Getafe in Madrid (Spain), between March 18, 2020 and April 18, 2020. Patients admitted to this unit had been diagnosed with COVID-19 (determined by a positive PCR result for SARS-CoV-2 in a nasopharyngeal exudate sample, and compatible symptoms; if the PCR was not available, diagnosis was based on symptoms with compatible laboratory and radiology data) and ARDS in accordance with the Berlin criteria [6]. Patients included in the analysis were between the ages of 18 and 80, who had $\mathrm{PaO}_{2} / \mathrm{FiO}_{2}<200 \mathrm{mmHg}$ or $\mathrm{SpO}_{2} / \mathrm{FiO}_{2}$ ratio $<240$, and who were treated with high-flow oxygen therapy through nasal cannula. Patients were excluded from the study if they presented immediate need for orotracheal intubation (OTI) due to hemodynamic instability, inability to protect the upper airway, or respiratory acidosis, as well as those with a DoNot-Intubate (DNI) order.

\section{Respiratory support modality}

The equipment used to deliver high-flow oxygen therapy was the AIRVO2 (Fisher \& Paykel Healthcare), with nasal cannula. Treatment was initiated with a programmed temperature between $31^{\circ} \mathrm{C}$ and $37^{\circ} \mathrm{C}$ according to tolerance, with high flows of $50-60$ $\mathrm{L} / \mathrm{min}$, and adjusting $\mathrm{FiO}_{2}$ to maintain $\mathrm{SatO}_{2}>92 \%$. Throughout the therapy, the patients were monitored with non-invasive mea- surement of heart rate, oxygen saturation and respiratory rate. All patients wore a surgical mask during high-flow therapy to reduce the risk of transmission of the virus through droplets or aerosols. When the situation allowed, $\mathrm{FiO}_{2}$ was initially reduced to 0.5 , then the flow was reduced until therapy was withdrawn. If the patient's status deteriorated, the approach was intubation and transfer to the ICU at the discretion of the responsible physician. In some cases, the pandemic situation led to the need to delay intubation because the number of beds in the intensive care unit were limited.

\section{Clinical variables}

The main study variable was the proportion of patients who required intubation at day 30, which was considered failed HFNC therapy. Data were collected for demographic variables, comorbidities, laboratory and clinical variables, including vital signs and ROX index [7] (ratio of pulse oximetry/fraction of inspired oxygen to respiratory rate) measured at two different times: prior to the start of high-flow therapy, and after 2 to $6 \mathrm{~h}$ of therapy. We also analysed the utility of the ROX index to predict the need for intubation.

\section{Statistical analysis}

Quantitative variables, expressed as mean \pm standard deviation, were compared using the Student's $t$-test. The qualitative variables were expressed in percentages and compared using either the chi-square test or Fisher's exact test. A uni/multivariate analysis was performed to evaluate the variables associated with intubation. The Kaplan-Meier method was used to obtain survival curves, and the different survival probabilities were compared using the logrank test. We explored the optimal cut-off point for the ROX index to predict failure of high-flow oxygen therapy by analysing diagnostic performance with ROC curves. The area under the curve was analysed for overall accuracy. A $p<0.05$ was considered significant. The statistical analysis was performed using STATA 14 software (Stata Corp. Stata Statistical Software: Release 14, College Station, TX, USA). This study has obtained a favourable report from the Ethics Committee for Research with Medicines (CEIm) of the Hospital Universitario de Getafe. Given the retrospective nature of the study, no informed consent was collected.

\section{Results}

Overall, 196 patients were admitted to our pulmonology unit from March 18 to April 18, 2020 with a diagnosis of acute respiratory infection due to COVID-19. A total of 40 patients with ARDS treated with HFNC were finally included in this study (Figure 1). Mean age was 58.9 years, and $70 \%$ were men. Twenty-one patients $(52.5 \%)$ experienced therapy failure and required intubation at day 30 , with a median time-to-intubation of two days (IQR: 1-4). The overall mortality rate was nine patients $(22.5 \%)$, all of whom were in the failed HFNC therapy group.

\section{Characteristics of patients with therapy failure}

The baseline characteristics of the patients, divided according to the need for intubation, are reflected in Table 1. The patients who did not require intubation had lower serum LDH levels and higher creatinine levels. They also presented lower values of $\mathrm{PCO}_{2}$ and bicarbonate in the arterial-blood gas test before starting HFNC.

\section{Respiratory variables}

The respiratory variables before initiating high-flow therapy and after 2 to $6 \mathrm{~h}$ of therapy are shown in Table 2. The $\mathrm{SpO}_{2} / \mathrm{FiO}_{2}$ ratio after starting HFNC was significantly higher in the group that did not require intubation $(113.4 \pm 6.6 v s 93.7 \pm 6.7 ; \mathrm{p}=0.020)$, as was the ROX index $(5.0 \pm 1.6$ vs $4.0 \pm 1.0 ; \mathrm{p}=0.018)$. The ROX index was 
shown to have good diagnostic performance in predicting the need for intubation, with an area under the ROC curve (AUC) of 0.712 . The optimal cut-off point was an ROX value of 4.94, measured 2 to $6 \mathrm{~h}$ after starting therapy. In the survival analysis, a ROX value of

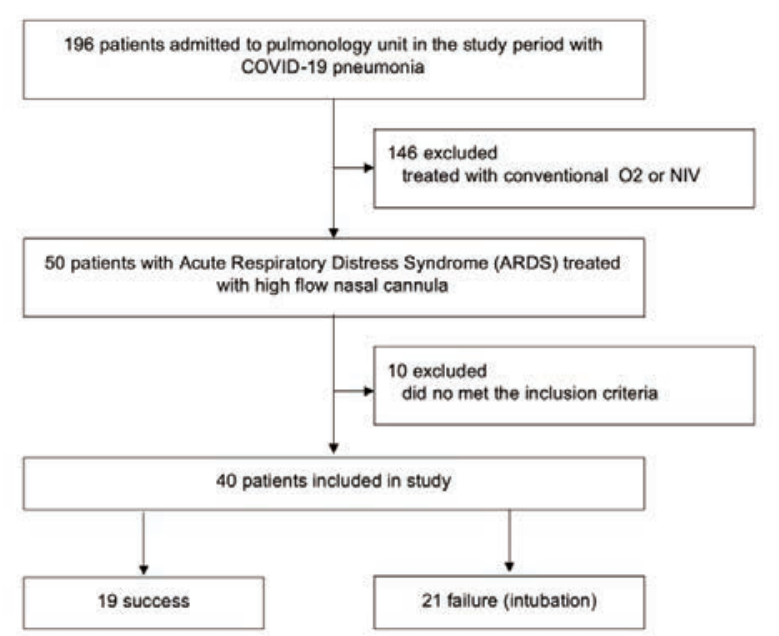

Figure 1. Flowchart of the study. less than 4.94 was associated with increased risk of intubation (HR 4.03 [95\% CI 1.18 - 13.7]; p=0.026). Figure 2 shows the KaplanMeier curve for the probability of patients with HFNC to remain off mechanical ventilation, according to the optimal ROX cut-off point.

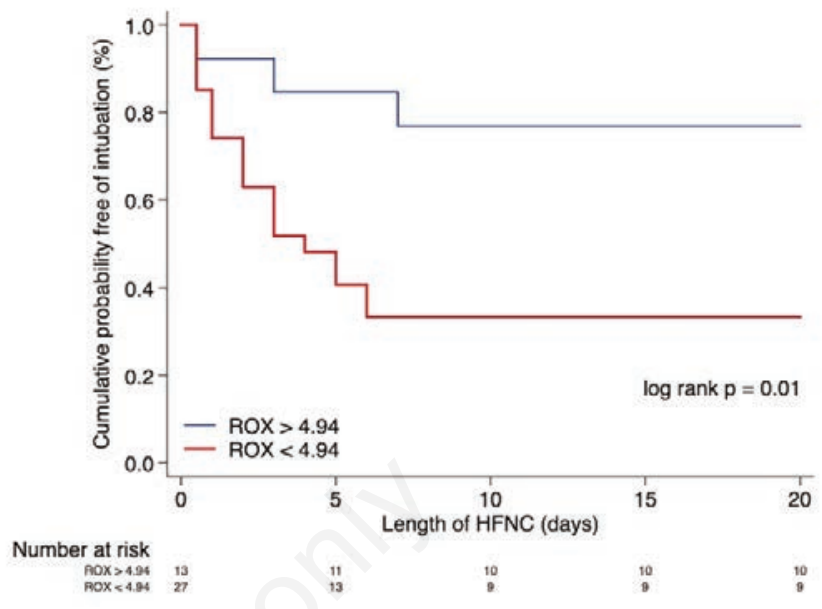

Figure 2. Kaplan-Meier curve showing the accumulated probability of remaining intubation-free in patients with high-flow nasal cannula, according to ROX index.

Table 1. Baseline characteristics and comparison between the two groups

\begin{tabular}{|c|c|c|c|c|}
\hline & $\begin{array}{c}\text { Total (\%) } \\
n=40\end{array}$ & $\begin{array}{c}\text { HFNC success } \\
n=19\end{array}$ & $\begin{array}{c}\text { HFNC failure } \\
n=21\end{array}$ & $\mathbf{p}$ \\
\hline Age (years) & $58.9(11.8)$ & $56.6(12.8)$ & $60.9(10.8)$ & 0.260 \\
\hline Gender (male) & $28(70 \%)$ & $14(74 \%)$ & $14(67 \%)$ & 0.740 \\
\hline BMI & $29.5(4.5)$ & $28.1(3.2)$ & $30.5(5.1)$ & 0.180 \\
\hline $\begin{array}{l}\text { Comorbidity } \\
\text { Hypertension } \\
\text { Dyslipidaemia } \\
\text { Diabetes } \\
\text { COPD } \\
\text { Asthma } \\
\text { OSAS } \\
\text { Coronary heart disease } \\
\text { Chronic kidney disease } \\
\text { Immunosuppression }\end{array}$ & $\begin{array}{c}16(40 \%) \\
13(32 \%) \\
8(20 \%) \\
2(5 \%) \\
5(12 \%) \\
5(12 \%) \\
2(5 \%) \\
3(8 \%) \\
4(10 \%)\end{array}$ & $\begin{array}{c}9(47 \%) \\
6(32 \%) \\
3(16 \%) \\
1(5 \%) \\
2(11 \%) \\
3(16 \%) \\
0 \\
3(16 \%) \\
2(11 \%)\end{array}$ & $\begin{array}{c}7(33 \%) \\
7(33 \%) \\
5(24 \%) \\
1(5 \%) \\
3(14 \%) \\
2(10 \%) \\
2(10 \%) \\
0 \\
2(10 \%)\end{array}$ & $\begin{array}{c}0.520 \\
1 \\
0.700 \\
1 \\
1 \\
0.650 \\
0.490 \\
0.980 \\
1\end{array}$ \\
\hline Charlson & $2(1.8)$ & $1.8(1.9)$ & $2.1(1.7)$ & 0.590 \\
\hline CURB65 & $1.2(0.9)$ & $1(0.7)$ & $1.3(1.0)$ & 0.250 \\
\hline SOFA score & $4.4(0.7)$ & $4.5(0.8)$ & $4.2(0.6)$ & 0.200 \\
\hline $\begin{array}{l}\text { Laboratory findings } \\
\text { Haemoglobin, g/dL } \\
\text { Lymphocyte count, } 10 * 3 / \mu \mathrm{L} \\
\text { Platelet count, } 10 * 3 / \mu \mathrm{L} \\
\text { D-dimer, } \mathrm{mg} / \mathrm{L} \\
\text { Creatinine, } \mathrm{mg} / \mathrm{dL} \\
\text { Lactate dehydrogenase, } \mathrm{U} / \mathrm{L} \\
\text { C-reactive protein, } \mathrm{mg} / \mathrm{dL} \\
\mathrm{pH} \\
\mathrm{PaCO}_{2}, \mathrm{mmHg} \\
\mathrm{PaO}_{2}, \mathrm{mmHg} \\
\text { Bicarbonate }\end{array}$ & $\begin{array}{c}13.7(1.1) \\
0.8(0.3) \\
286.2(114.4) \\
5.7(10.8) \\
0.9(0.3) \\
520(165) \\
119.6(99.6) \\
7.41(0.1) \\
37.1(8.9) \\
68.8(17.8) \\
23.4(4.3)\end{array}$ & $\begin{array}{c}13.7(1.1) \\
0.9(0.3) \\
282.6(127) \\
6.2(14.4) \\
0.9(0.3) \\
443.9(97.4) \\
128.3(100.6) \\
7.42(0.0) \\
31.4(3.3) \\
67.2(14.0) \\
21.5(3.8)\end{array}$ & $\begin{array}{c}13.6(1.1) \\
0.7(0.4) \\
289(104) \\
5.1(6.0) \\
0.8(0.2) \\
588(184.9) \\
111.8(100.6) \\
7.39(0.1) \\
42.7(9.2) \\
70.4(21.5) \\
25.3(4.0)\end{array}$ & $\begin{array}{l}0.790 \\
0.250 \\
0.850 \\
0.760 \\
0.042 \\
0.004 \\
0.610 \\
0.055 \\
0.001 \\
0.680 \\
0.035\end{array}$ \\
\hline Days HFNC (median, IQR) & $3(1-5)$ & $6(5-8)$ & $2(1-4)$ & 0.010 \\
\hline Death rate & $9(22.5 \%)$ & 0 & $9(42.8 \%)$ & 0.020 \\
\hline
\end{tabular}

Data are means \pm SD, median (IQR) or n (\%). HFNC, high-flow nasal cannula; BMI, body-mass index is the weight in kilograms divided by the square of the height in meters; COPD, chronic obstructive

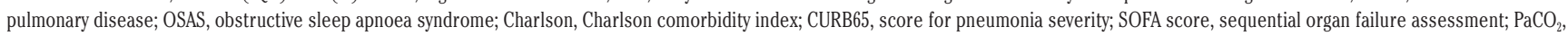
partial pressure of arterial carbon dioxide; $\mathrm{PaO}_{2}$, partial pressure of arterial oxygen. 


\section{Discussion}

Our study demonstrates that HFNC is a useful therapy for the management of patients with ARDS secondary to COVID-19. The main finding is that HFNC enabled us to treat ARDS successfully in a high proportion of patients $(47.5 \%)$ without requiring invasive ventilatory support and with low mortality. Therefore, the use of HFNC may be the first treatment option for ARDS due to its efficacy in avoiding intubation and associated complications.

During the COVID-19 pandemic, health resources have been limited by the imbalance caused by high demand. Based on our results, the strategy of initiating ARDS treatment with HFNC is useful, safe and seems reasonable for optimizing the resources available in order to avoid admissions to intensive care units or to limit them only to the most severe patients.

Additionally, we have observed that the ROX index performs well in determining which patients will need intubation after initiating HFNC. A ROX index less than 4.94 measured 2 to 6 hours after initiation of therapy was associated with increased risk of intubation (HR 4.03 [95\% CI 1.18-13.7]; $p=0.026$ ). Therefore, it is a good tool for identifying patients in whom therapy may fail and are therefore at high risk of requiring intubation. These patients may benefit from closer monitoring or early intubation. A recurring reason for concern in ARDS is the finding of higher mortality in patients with late intubation [8], which has brought into question the usefulness of non-invasive respiratory support. Delayed intubation can increase mortality, so it is important to use this therapy in a safe environment, with continuous monitoring of patient response to treatment. Also, it is necessary to have tools that help predict in which individuals this therapy will fail so as not to delay intubation. In this context, some studies support the use of the ROX index. An observational study in 2015 [9] showed that an elevated respiratory rate and a low $\mathrm{PaO}_{2} / \mathrm{FiO}_{2}$ was present in patients in whom therapy failed. Subsequently, Roca et al. [10] validated the ROX index in the first hours of respiratory support as a predictor of HFNC success in avoiding intubation. In their paper, an index higher than 4.88 after $12 \mathrm{~h}$ of treatment predicted a lower risk of intubation. Our results are comparable, since our optimal cut-off point was slightly higher at 4.94. A further relevant finding is the observation of increased levels of $\mathrm{PaCO}_{2}$ and $\mathrm{HCO}_{3}$, prior to the beginning of the respiratory support, in those patients with no response to HFNC. The increased levels of $\mathrm{PaCO}_{2}$ suggest the pre-

Table 2. Respiratory variables before treatment with high-flow nasal cannula.

\begin{tabular}{lccc} 
& $\begin{array}{c}\text { HFNC success } \\
\mathbf{n = 1 9}\end{array}$ & $\begin{array}{c}\text { HFNC failure } \\
\mathbf{n = 2 1}\end{array}$ & $\mathbf{p}$ \\
\hline Baseline & & & \\
Heart rate (bpm) & $79.0(10.1)$ & $84.9(11.6)$ & 0.099 \\
Respiratory rate (rpm) & $28.1(8.1)$ & $30.4(12.1)$ & 0.490 \\
\hline $\mathrm{SpO}_{2} / \mathrm{FiO}_{2}$ & $103.0(3.4)$ & $101.4(5.1)$ & 0.250 \\
$\mathrm{ROX}_{\text {index }}$ & $4.0(1.4)$ & $3.7(1.0)$ & 0.560 \\
\hline 2-6 h of HFNC & & & \\
Heart rate (bpm) & $74.6(14.0)$ & $88.4(20.9)$ & 0.026 \\
Respiratory rate (rpm) & $23.6(7.0)$ & $26.0(6.7)$ & 0.290 \\
\hline $\mathrm{SpO}_{2}$ FiO & $113.4(36.6)$ & $93.7(6.7)$ & 0.020 \\
$\mathrm{ROX}_{2}$ index & $5.0(1.6)$ & $4.0(1.0)$ & 0.018 \\
\hline
\end{tabular}

Data are means \pm SD. Baseline treatment was standard oxygen therapy given through a nonrebreather face mask at a flow rate of 15 litres/min; HFNC, high-flow nasal cannula; $\mathrm{SpO}_{2} / \mathrm{Fi}$, pulse oximetry; $\mathrm{FiO}_{2}$, fraction of inspired oxygen; $\mathrm{ROX}$ index, $\mathrm{SpO}_{2} / \mathrm{FiO}_{2}$ :respiratory rate. sence of higher muscle fatigue due to the inspiratory workload. This would be indicative of a ventilatory failure and thus, a more severe clinical status [11].

On the other hand, increased $\mathrm{HCO}_{3}$ indicates a renal response against the respiratory acidosis which usually takes a few days to develop, and may suggest a longer evolution of the respiratory condition. Summarizing, we can conclude that the respiratory failure severity, as determined by the blood gas analysis, may explain the HFCN failure in our patients. The HFNC provides ventilatory assistance and has proven to be an useful therapy for respiratory failure [12]. Nevertheless, our findings stress the importance of using cautiously, and closely monitor, the response in those patients with ARDS who develop analytical signs of respiratory failure.

Despite our good results, there are scarcely any studies that describe the efficacy of HFNC in patients with COVID-19. The only study published to date provides the results from 17 patients treated with HFNC for ARDS due to COVID-19.13 The authors report a $41 \%$ rate of therapy failure, which is similar to our results. In another study published during the pandemic including 52 critically ill patients with SARS-CoV-2 pneumonia [14], 71\% required intubation. In this cohort, a total of 33 patients received high-flow oxygen therapy, $51.5 \%$ of whom survived, but no mention was made of whether they required later intubation.

One of the reasons why there are few studies with data on the efficacy of HFNC is because of safety warnings. The risk of contagion for healthcare workers due to the high dispersion of particles has led some authors to discourage its use [15]. However, during the 2003 SARS epidemic, healthcare workers exposed to highflow therapy did not present increased risk of developing the disease [16]. Subsequently, a recent review by Li et al. [17] observed that the risk of dispersion was similar to that of conventional oxygen masks, and transmission was reduced when a surgical mask was used over the nasal cannula.

The efficacy of this therapy has previously shown favourable results in ARDS secondary to other viral infections. In influenza virus infections (subtype H1N1) [18], an intubation rate of 55\% was observed, which is similar to the results of our study. Data from the FLORALI cohort [3] (a 2015 RCT of patients with hypoxemic respiratory failure, including 105 patients treated with HFNC) indicated a lower intubation rate of $38 \%$, although it is true that no all the patients met ARDS criteria and acute respiratory failure was secondary to various aetiologies. When compared to standard oxygen therapy and NIV, HFNC presented better results for the intubation rate of patients with $\mathrm{PaO}_{2} / \mathrm{FiO}_{2} \leq 200 \mathrm{mmHg}$. Other observational studies with HFNC [9] have shown intubation rates of $40 \%$ in the treatment of ARDS, and also better tolerance than the NIV interface. The patients in our study had a mean treatment of three days (six in the group that did not require intubation, and two in the patients who were finally intubated), which demonstrates that one of the factors for success of this therapy is good patient tolerance.

A remarkable observation in our study has been the low mortality of our cohort, with only $22.5 \%$ mortality despite all patients meeting ARDS criteria. These results are better than those reported in other studies, where mortality ranged between $52 \%$ and $61 \%$ $[14,19]$. In the largest series published to date of 33 patients with COVID-19 treated with HFNC [14], the mortality rate was $49 \%$. Different reasons could explain these results. Firstly, all the patients were treated in a highly specialised intermediate respiratory care unit with expert staff $24 \mathrm{~h}$ a day and equipped with continuous monitoring, in which semi-critical care was administered. Furthermore, our population presents a lower risk profile compared to other studies. The mean CURB65 score at admission was 1.2 , which stratified the 30-day risk of mortality between $2.7 \%$ and $6.8 \%$ [20]. In addition, patients for whom ICU admission was con- 
sidered futile were excluded from the analysis, meaning that highflow therapy had been their therapeutic ceiling.

The main limitation of this study is the small number of patients included, since the data was collected from a single centre. Being a retrospective study, the criteria for intubation of the patients were not defined. However, this analysis provides information about the experience of high-flow nasal cannula oxygen therapy in critically ill patients with COVID-19 and its applicability in other centres. In conclusion, high-flow oxygen therapy can be a useful treatment for ARDS due to COVID-19, both as respiratory support alone or as a bridge therapy to OTI in a pandemic situation. When administered in a unit monitored by an expert staff, HFNC therapy can avoid intubation or delay admission to an intensive care unit, without increasing overall mortality secondary to delayed intubation.

\section{Acknowledgements}

We thank the health professionals from the Hospital Universitario de Getafe, Madrid.

\section{References}

1. Zhu N, Zhang D, Wang W, Li X, Yang B, Song J, et al. A novel coronavirus from patients with pneumonia in China, 2019. N Engl J Med 2020;382:727-33.

2. Wang D, Hu B, Hu C, Zhu F, Liu X, Zhang J, et al. Clinical characteristics of 138 hospitalized patients with 2019 novel coronavirus-infected pneumonia in Wuhan, China. JAMA 2020;323:1061.

3. Frat JP, Thille AW, Mercat A, Girault C, Ragot S, Perbet S, et al. High-flow oxygen through nasal cannula in acute hypoxemic respiratory failure. N Engl J Med 2015;372:2185-96.

4. Parke RL, Eccleston ML, McGuinness SP. The Effects of flow on airway pressure during nasal high-flow oxygen therapy. Respir Care 2011;56:1151-5.

5. Rochwerg B, Granton D, Wang DX, Helviz Y, Einav S, Frat JP, et al. High flow nasal cannula compared with conventional oxygen therapy for acute hypoxemic respiratory failure: a systematic review and meta-analysis. Intensive Care Med 2019;45:563-72.

6. ARDS Definition Task Force, Ranieri VM, Rubenfeld GD, Thompson BT, Ferguson ND, Caldwell E, et al. Acute respiratory distress syndrome: the Berlin definition. JAMA 2012;307:2526-33.

7. Roca O, Messika J, Caralt B, García-de-Acilu M, Sztrymf B,
Ricard J-D, et al. Predicting success of high-flow nasal cannula in pneumonia patients with hypoxemic respiratory failure: The utility of the ROX index. J Crit Care 2016;35:200-5.

8. Kang BJ, Koh Y, Lim CM, Huh JW, Baek S, Han M, et al. Failure of high-flow nasal cannula therapy may delay intubation and increase mortality. Intensive Care Med 2015;41:62332.

9. Messika J, Ahmed K Ben, Gaudry S, Miguel-Montanes R, Rafat C, Sztrymf B, et al. Use of high-flow nasal cannula oxygen therapy in subjects with ARDS: A 1-year observational study. Respir Care 2015;60:162-9.

10. Roca O, Caralt B, Messika J, Samper M, Sztrymf B, Hernández $\mathrm{G}$, et al. An index combining respiratory rate and oxygenation to predict outcome of nasal high-flow therapy. Am J Respir Crit Care Med 2019;199:1368-76.

11. Roussos C, Koutsoukou A. Respiratory failure. Eur Respir J 2003;47:s3-14.

12. Nishimura M. High-flow nasal cannula oxygen therapy devices. Respir Care 2019;64:735-42.

13. Wang K, Zhao W, Li J, Shu W, Duan J. The experience of highflow nasal cannula in hospitalized patients with 2019 novel coronavirus-infected pneumonia in two hospitals of Chongqing, China. Ann Intensive Care 2020;10:37.

14. Yang X, Yu Y, Xu J, Shu H, Xia J, Liu H, et al. Clinical course and outcomes of critically ill patients with SARS-CoV-2 pneumonia in Wuhan, China: a single-centered, retrospective, observational study. Lancet Respir Med 2020;8:475-81.

15. Cheung JCH, Ho LT, Cheng JV, Cham EYK, Lam KN. Staff safety during emergency airway management for COVID-19 in Hong Kong. Lancet Respir Med. 2020;8:e19.

16. Raboud J, Shigayeva A, McGeer A, Bontovics E, Chapman M, Gravel D, et al. Risk factors for SARS Transmission from patients requiring intubation: Amulticentre investigation in Toronto, Canada. PLoS One 2010;5:e10717.

17. Li J, Fink JB, Ehrmann S. High-flow nasal cannula for COVID-19 patients: low risk of bio-aerosol dispersion. Eur Respir J 2020;55:2000892.

18. Rello J, Pérez M, Roca O, Poulakou G, Souto J, Laborda C, et al. High-flow nasal therapy in adults with severe acute respiratory infection: A cohort study in patients with 2009 influenza A/H1N1v. J Crit Care 2012;27:434-9.

19. Wu C, Chen X, Cai Y, Xia J, Zhou X, Xu S, et al. Risk factors associated with acute respiratory distress syndrome and death in patients with coronavirus disease 2019 pneumonia in Wuhan, China. JAMA Intern Med 2020;180:e200994.

20. Lim WS, Eerden MM van der, Laing R, Boersma WG, Karalus $\mathrm{N}$, Town GI, et al. Defining community-acquired pneumonia severity on presentation to hospital: an international derivation and validation study. Thorax 2003;58:377-82.

Received for publication: 21 June 2020. Accepted for publication: 27 August 2020.

This work is licensed under a Creative Commons Attribution-NonCommercial 4.0 International License (CC BY-NC 4.0).

CCopyright: the Author(s), 2020

Licensee PAGEPress, Italy

Multidisciplinary Respiratory Medicine 2020; 15:693

doi:10.4081/mrm.2020.693 